\title{
ON CONTINUITY OF SYMMETRIC RESTRICTIONS OF BOREL FUNCTIONS
}

\author{
MICHAL MORAYNE
}

\begin{abstract}
We prove that if $X$ is a complete metric space dense-in-itself, $Y$ is a compact metric space and $F: X \times X \backslash\{(x, x): x \in X\} \rightarrow Y$ is a Borel-measurable function such that $F\left(x_{1}, x_{2}\right)=F\left(x_{2}, x_{1}\right)$ for every $x_{1}, x_{2} \in X, x_{1} \neq x_{2}$, then there is a perfect subset $P$ of $X$ such that $F$ is uniformly continuous on $P \times P \backslash\{(x, x)$ : $x \in P\}$. An immediate corollary of the above result is the following theorem proved by Bruckner, Ceder and Weiss: If $F$ is a real continuous function defined on a perfect set $P \subset R$, there is a perfect subset $Q$ of $P$ such that $f \mid Q$ has a derivative (finite or infinite) at every point of $Q$.
\end{abstract}

For any set $A$ we shall use the notation $A^{2}=A \times A$ and $D(A)=\{(x, x)$ : $x \in A\} . \delta(A)$ will denote the diameter of $A$ for $A \subset R$. A subset $B$ of a cartesian square is called symmetric if $(y, x) \in B$ whenever $(x, y) \in B$.

We prove the following

THEOREM. If $X$ is a complete metric space dense-in-itself, $Y$ is a compact metric space, and $F: X^{2} \backslash D(X) \rightarrow Y$ is a Borel-measurable function such that $F\left(x_{1}, x_{2}\right)=$ $F\left(x_{2}, x_{1}\right)$ for every $x_{1}, x_{2} \in X, x_{1} \neq x_{2}$, then there is a perfect (i.e., nonempty, closed, and dense-in-itself ) subset $P$ of $X$ such that $F$ is uniformly continuous on $P^{2} \backslash D(P)$, whence it has a continuous extension to the whole $P^{2}$.

The first part of the conclusion of this theorem, with "continuity" instead of "uniform continuity", is 2.3 in [2]. But, in fact, we want the continuous extension of $F$ to $D(P)$ (what uniform continuity gives us), whence we can derive the following result of Bruckner, Ceder, and Weiss [1]:

Let $f$ be a real continuous function on a perfect set $P$ of real numbers. Then there exists a perfect set $Q$ contained in $P$ such that the restriction of $f$ to $Q$ has a derivative ( finite or infinite) at every point of $Q$.

Indeed it is enough to put $X=P, Y=[0,1]$ and

$$
F\left(x_{1}, x_{2}\right)=\varphi\left(\left(f\left(x_{1}\right)-f\left(x_{2}\right)\right) /\left(x_{1}-x_{2}\right)\right) \text { for } x_{1} \neq x_{2},
$$

where $\varphi$ is any homeomorphism of $R$ onto $(0,1)$.

From now on $\mathscr{C}$ will denote only the Cantor discontinuum, i.e., the set of all numbers of $[0,1]$ with triadic expansions in which digit one does not occur. 
We can assume without any loss of generality that $X=\mathscr{C}$, because every complete dense-in-itself metric space contains a copy of $\mathscr{C}$.

We can also assume $Y=\mathscr{C}$. Indeed, for every compact metric space $Y$ there is a continuous surjection $f: \mathscr{C} \rightarrow Y$. Then $g(y)=\min \{c \in \mathscr{C}: f(c)=y\}$ is a Borel measurable function such that $f \circ g$ is the identity map on $Y$. Thus, if $F$ satisfies the assumptions of our theorem, we can consider $g \circ F: X^{2} \backslash D(X) \rightarrow \mathscr{C}$, for which, if the theorem is true for $\mathscr{C}$ as an image space, there is a perfect set $P \subset X$ such that $g \circ F$ is uniformly continuous on $P^{2} \backslash D(P)$. Now it is enough to notice that $F=f \circ g \circ F$.

Our main tool is the following result of Galvin (announced in [3 and 4] in more general form):

Main Lemma. Let $A$ and $B$ be symmetric Borel subsets of $\mathscr{C}^{2}$ and $\mathscr{C}^{2} \backslash D(\mathscr{C})$ $\subset A \cup B$. Then there exists a perfect subset $P$ of $\mathscr{C}$ such that $P^{2} \backslash D(P) \subset A$ or $P^{2} \backslash D(P) \subset B$.

The proof of the lemma can be found in [2]. It was based on Mycielski's theorem of [5] (2.2 in [2]).

Proof OF THEOREM. We can assume with no loss of generality that $F$ is continuous on $\mathscr{C}^{2} \backslash D(\mathscr{C})$ (see 2.3 in [2]).

By the Main Lemma there exists a perfect set $Q \subset \mathscr{C}$ such that $F\left(Q^{2} \backslash D(Q)\right) \subset[0$, $\left.\frac{1}{2}\right]$ or $F\left(Q^{2} \backslash D(Q)\right) \subset\left[\frac{1}{2}, 1\right]$. Assume $F\left(Q^{2} \backslash D(Q)\right) \subset\left[0, \frac{1}{2}\right]$. Let $P_{(0)}, P_{(1)}$ be disjoint perfect sets contained in $Q$ and $\delta\left(P_{(i)}\right)<1, i=0,1$.

Again by the Main Lemma we can find disjoint perfect sets $P_{(i, 0)}, P_{(i, 1)} \subset P_{(i)}$, $i=0,1$, such that $F\left(P_{(i, j)}^{2} \backslash D\left(P_{(i, j)}\right)\right) \subset\left[0, \frac{1}{4}\right]$ or $F\left(P_{(i, j)}^{2} \backslash D\left(P_{(i, j)}\right)\right) \subset\left[\frac{1}{4}, \frac{1}{2}\right]$ and $\delta\left(P_{(i, j)}\right)<\frac{1}{2}$.

Continuing the choice of perfect sets $P_{\left(i_{1}, \ldots, i_{n}\right)}$ as above, we obtain $P_{\left(i_{1}, \ldots, i_{n+1}\right)} \subset$ $P_{\left(i_{1}, \ldots, i_{n}\right)}, P_{\left(i_{1}, \ldots, i_{n}, 0\right)} \cap P_{\left(i_{1}, \ldots, i_{n}, 1\right)}=\varnothing, \delta\left(P_{\left(i_{1}, \ldots, i_{n}\right)}\right)<1 / n$. Moreover,

$F\left(P_{\left(i_{1} \ldots, i_{n}\right)}^{2} \backslash D\left(P_{\left(i_{1}, \ldots, i_{n}\right)}\right)\right) \subset\left[\sum_{j=1}^{n} \alpha_{j} 2^{-j}, 2^{-n}+\sum_{j=1}^{n} \alpha_{j} 2^{-j}\right]$ for some $\alpha_{j}=0$ or 1.

We shall prove that the set $P=\bigcap_{n=1} \bigcup_{\left(i_{1}, \ldots, i_{n}\right)} P_{\left(i_{1}, \ldots, i_{n}\right)}$ has the desired property, i.e. $F \mid P^{2} \backslash D(P)$ is uniformly continuous.

Let us fix $n$. Let

$$
A=\bigcup\left\{P_{\left(i_{1}, \ldots, i_{n}\right)} \times P_{\left(j_{1}, \ldots, j_{n}\right)}:\left(i_{1}, \ldots, i_{n}\right) \neq\left(j_{1}, \ldots, j_{n}\right)\right\} .
$$

$A$ is compact, so $F \mid A$ is uniformly continuous, whence there is $\delta_{0}>0$ such that $|F(u)-F(v)|<2^{-n}$ whenever $u, v \in A$ and the distance from $u$ to $v$ is less than $\delta_{0}$.

Let $\delta_{1}$ be the smallest distance between different sets $P_{\left(i_{1}, \ldots, i_{n}\right)} \times P_{\left(j_{1}, \ldots, j_{n}\right)}$.

The oscillation of $F$ on each set $P_{\left(i_{1}, \ldots, i_{n}\right)}^{2} \backslash D\left(P_{\left(i_{1}, \ldots, i_{n}\right)}\right)$ does not exceed $2^{-n}$. Thus, $|F(u)-F(v)| \leqslant 2^{-n}$ whenever $u, v \in P^{2} \backslash D(P)$ and the distance from $u$ to $v$ is less than $\min \left\{\delta_{0}, \delta_{1}\right\}$. This finishes the proof.

I am very grateful to Professor Czesław Ryll-Nardzewski for his advice and suggestions. 
My thanks are also due to Rysiek Komorowski and Janusz Pawlikowski for their remarks concerning this paper.

\section{REFERENCES}

1. A. M. Bruckner, J. G. Ceder and M. L. Weiss, On differentiability structure of real functions, Trans. Amer. Math. Soc. 142 (1969), 1-13.

2. J. P. Burgess, $A$ selector principle for $\Sigma_{1}^{1}$ equivalence relations, Michigan Math. J. 24 (1977), 65-76.

3. F. Galvin, Partition theorems for the real line, Notices Amer. Math. Soc. 15 (1968), 660.

4. __ Errata to "Partition theorems for the real line", Notices Amer. Math. Soc. 16 (1969), 1095.

5. J. Mycielski, Independent sets in topological algebras, Fund. Math. 55 (1964), 139-147

Instytut Matematyczny, UniWersytet Wrockawski, Pl. GrunWaldzki 2 / 4, 50 - 384 Wrockaw, POLAND 